\title{
Commonalities in Metabolism of Arsenicals
}

\author{
Blakely M. Adair, ${ }^{\mathrm{A}}$ Stephen B. Waters, ${ }^{\mathrm{B}}$ Vicenta Devesa,${ }^{\mathrm{C}}$ Zuzana Drobna, ${ }^{\mathrm{D}}$ \\ Miroslav Styblo, ${ }^{\mathrm{C}, \mathrm{D}}$ and David J. Thomas ${ }^{\mathrm{A}, \mathrm{E}}$ \\ A Experimental Toxicology Division, National Health and Environmental Effects Research Laboratory, \\ US Environmental Protection Agency, Research Triangle Park, NC 27711, USA. \\ B Curriculum in Toxicology, University of North Carolina, Chapel Hill, NC 27599, USA. \\ C Center for Environmental Medicine, Asthma, and Lung Biology, University of North Carolina, \\ Chapel Hill, NC 27599, USA. \\ D Department of Nutrition, School of Public Health, University of North Carolina, Chapel Hill, \\ NC 27599, USA. \\ ${ }^{\mathrm{E}}$ Corresponding author. Email: thomas.david@epamail.epa.gov
}

\begin{abstract}
Environmental Context. Health effects associated with inorganic arsenic include various cancers and increased risk of diabetes. Millions of people in Bangladesh and India are at risk through use of contaminated drinking water. When humans ingest inorganic arsenic, it is rapidly converted to methylated metabolites. Although this methylation process is largely understood, the metabolism of other arsenicals (e.g. arsenosugars to dimethylarsenic) is very unclear. Connections among pathways for metabolism of various arsenicals are now being elucidated. Commonalities and differences in these pathways may be important determinants of the risk associated with exposure to these agents.
\end{abstract}

\begin{abstract}
Elucidating the pathway of inorganic arsenic metabolism shows that some of methylated arsenicals formed as intermediates and products are reactive and toxic species. Hence, methylated arsenicals likely mediate at least some of the toxic and carcinogenic effects associated with exposure to arsenic. Trimethylarsonium compounds and arsenosugars are two other classes of arsenicals to which humans are routinely exposed and there is evidence that both classes are metabolized to produce methylated arsenicals. Here, we review evidence for production of methylated metabolism and consider the challenges posed in unraveling a complex web for metabolism of arsenicals in humans.
\end{abstract}

Keywords. arsenic — bioavailability — contaminant uptake — metabolism-affecting agents

Manuscript received: 7 July 2005.

Final version: 19 August 2005.

\section{Introduction}

Chronic exposure to inorganic arsenic (iAs) is associated with many adverse health effects, including increased risk of various cancers, vascular disease, and other effects such as increased risk of diabetes mellitus. ${ }^{[1]}$ Exposure to high levels of iAs as a contaminant of drinking water occurs worldwide with disastrous effects. ${ }^{[2]}$ Tens of millions of residents of Bangladesh and West Bengal, India, who use iAs-contaminated water may be the 'largest poisoning of a population in history'. [3] Critical factors in assessing the public health impact of iAs exposure are evaluating doseresponse relations and determining all sources of exposure. Besides iAs, other significant sources of arsenic exposure which warrant consideration in estimating dose include trimethylarsonium compounds (arsenobetaine and arsenocholine) and arsenosugars that are natural constituents of many organisms used as human food sources. Arsenobetaine, arsenocholine, and arsenosugars are present at high (ppm) levels in foods and are metabolized by humans to produce some of the methylated metabolites that are also produced after iAs ingestion. Thus, both unique and common metabolites can be identified for each class of arsenicals (Fig. 1). This review examines metabolism of iAs, trimethylarsonium compounds, and arsenosugars with emphasis on identifying common metabolites. New information about metabolism of these arsenicals will provide insights into their modes of action as toxins and carcinogens and poses some interesting analytical challenges. Meeting these challenges will permit a more comprehensive understanding of the consequences of exposure to these arsenicals.

\section{Inorganic Arsenic}

The pathway for iAs metabolism involves alternating steps of reduction of $\mathrm{As}^{\mathrm{V}}$ to $\mathrm{As}^{\mathrm{III}}$ and oxidative methylation of $\mathrm{As}^{\mathrm{III}}$ (Fig. 2). These reactions are enzymatically catalyzed. Glyceraldehyde-3-phosphate dehydrogenase reduces 


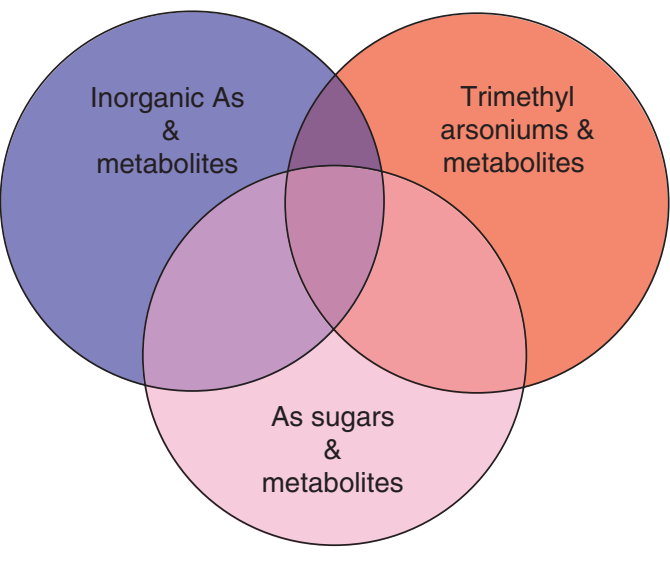

Fig. 1. Venn diagram for exposure to common and unique metabolites formed from inorganic arsenic, trimethylarsonium compounds, and arsenosugars. Common metabolites produced from each class of arsenical should be equivalent in terms of overall exposure and contribution to risk. arsenate to arsenite in human red blood cells. ${ }^{[4,5]}$ Arsenic(III) methyltransferase (AS3MT) catalyzes each subsequent step in this pathway, producing the products methylarsonic acid through trimethylarsine (Fig. 2). ${ }^{[6,7]}$ In human uroepithelial cells that neither express AS3MT nor methylate iAs, heterologous expression of recombinant rat AS3MT confers methylation capacity and modulates cytotoxicity of arsenicals. ${ }^{[8]}$ Intermediates and products in this pathway differ in reactivity and toxicity. In general, methylated arsenicals containing As ${ }^{\mathrm{III}}$ are most reactive, exceeding arsenite in cytotoxic and genotoxic potency. In addition, two methylated arsenicals containing $\mathrm{As}^{\mathrm{V}}$ (dimethylarsinic acid and trimethylarsine oxide) are complete carcinogens in rats. ${ }^{[9,10]}$ Hence, the conversion of iAs to methylated metabolites is an activation process ${ }^{[11]}$ and methylated arsenicals derived from the metabolism of other arsenicals should be considered in assessing the risk associated with exposure to these arsenicals.
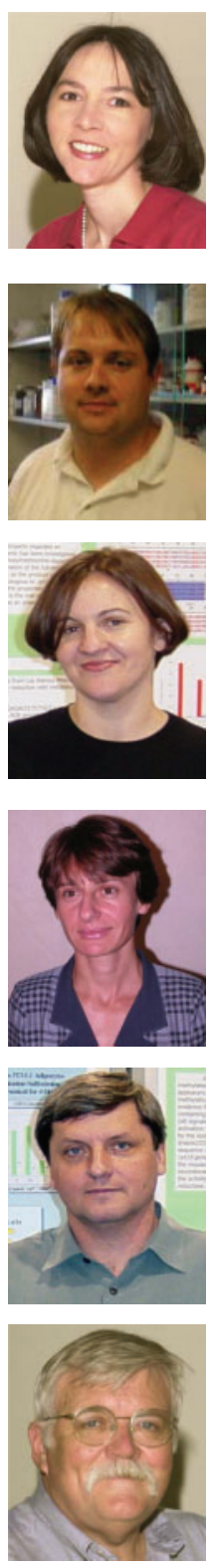

Blakely M. Adair received a Ph.D. in environmental toxicology from Texas Tech University in 2002. Since then she has been a postdoctoral fellow at the U.S. Environmental Protection Agency working with David Thomas. Her research focuses on the environmental analytical chemistry of toxicologically relevant contaminants, including method development for quantification of arsenicals in biological samples.

Stephen B. Waters received a Ph.D. in molecular biology from Wake Forest University School of Medicine in 2001. He was a postdoctoral fellow in the Curriculum in Toxicology at the University of North Carolina-Chapel Hill from 2001 until 2004 and worked with David Thomas. He is currently studying TAT-mediated protein transduction in the Department of Cardiology at the University of Illinois-Chicago.

Vicenta Devesa received a Ph.D. in analytical chemistry in 2002 from the University of Valencia. From 2003 to 2005 she held a MECD/Fulbright Postdoctoral Fellowship at the University of North Carolina at Chapel Hill and worked with Miroslav Styblo. Her research interests include speciation of arsenicals in biological samples. She is currently affiliated with the Institute of Agrochemistry and Food Technology, Burjassot, Valencia, Spain.

Zuzana Drobna received a Ph.D. in biochemistry from SlovakAcademy of Sciences in Bratislava in 1997. During graduate studies she worked at the Max Delbruck Institute in Berlin. She has been a postdoctoral fellow with Miroslav Styblo since 2001. Her research investigates the role of arsenic methylation in modulation of its toxic and cancer promoting effects in mammalian cells.

Miroslav Styblo is Research Associate Professor in the Department of Nutrition, University of North Carolina at Chapel Hill. His background and interests are in biochemical toxicology and nutritional biochemistry. Current research focuses on the metabolism and molecular toxicity of arsenic, on identification of biomarkers for exposure and chronic toxicity of arsenic, and on interactions between arsenic and essential metals and metalloids.

David J. Thomas is Research Toxicologist in the Experimental Toxicology Division of the National Health and Environmental Effects Research Laboratory of the U.S. Environmental Protection Agency. He is interested in the metabolism of metals and metalloids and in development of novel methods to identify metals and metalloids in biological matrices. 


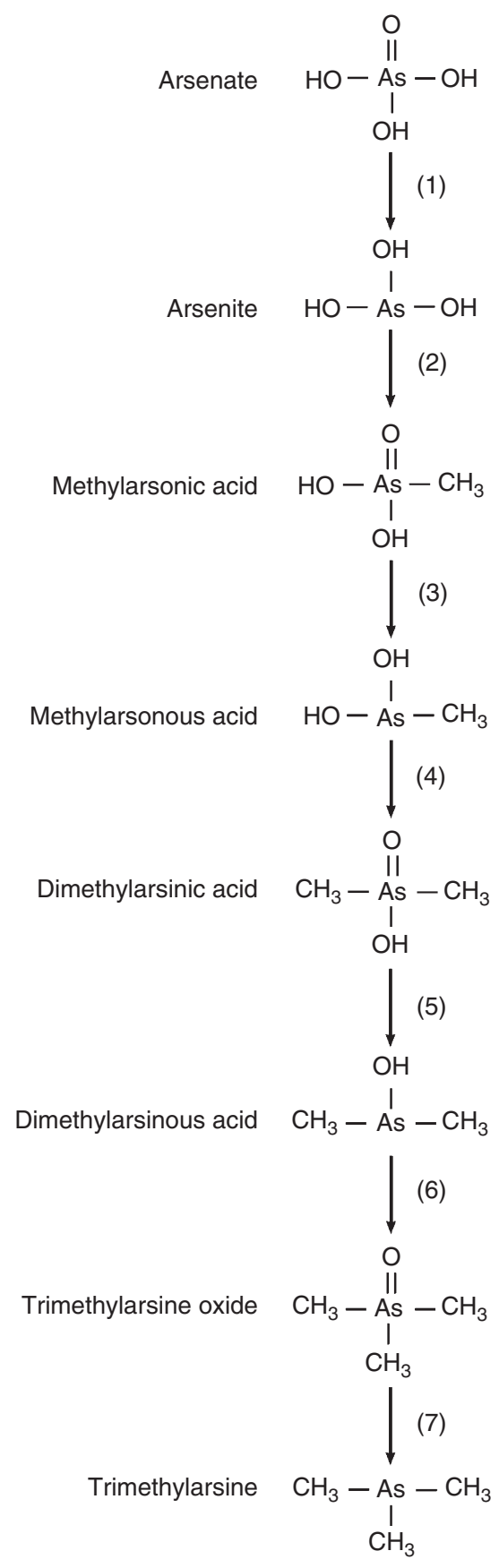

Fig. 2. Scheme for the conversion of inorganic arsenic to methylated metabolites. Reactions (1), (3), (5), and (7) convert $\mathrm{As}^{\mathrm{V}}$ to $\mathrm{As}{ }^{\mathrm{III}}$ and Reactions (2), (4), and (6) are oxidation methylation reactions.

\section{Trimethylarsonium Compounds}

Origins or functions of trimethylarsonium compounds (arsenocholine and arsenobetaine), which are found in many organisms from marine, freshwater, and terrestrial environments, are not well understood. Synthesis of arsenobetaine from a lyzed cell extract of Pseudomonas species suggests prokaryotes could produce trimethylarsonium compounds found in many marine organisms. ${ }^{[12]}$ Arsenobetaine could be an osmolyte for marine organisms living in hyperosmotic environments. ${ }^{[13,14]}$ Bacterial degradation of arsenobetaine to methylated arsenicals through formation of dimethylarsinoylacetate has been demonstrated in Paenibacillus and Pseudomonas species derived from Mytilus edulis, a marine mussel. ${ }^{[15]}$ These results suggest that microorganisms associated with eukaryotes (e.g. microflora of the gastrointestinal tract) could be critical to metabolism of trimethylarsonium compounds in higher organisms. In vitro conversion of arsenocholine to arsenobetaine by rat liver was demonstrated decades ago. ${ }^{[16]}$ Arsenobetaine administered orally or intravenously to rats, mice, or rabbits is rapidly excreted in urine with little, if any, metabolism. ${ }^{[17]}$ Another study in these species found arsenocholine was quickly converted to arsenobetaine without conversion to methylated metabolites. ${ }^{[18]}$ However, the relatively insensitive analytical methods used in these studies (ion-exchange chromatography and paper electrophoresis) might limit quantitation of methylated metabolites present at low levels in urine. A study of volunteers who consumed arsenobetaine-rich seafood found high levels of dimethylarsenic in urine using IEC-HG-ICP MS for speciation. ${ }^{[19]}$ Arsenocholine is converted in vitro to arsenobetaine and methylated metabolites. In cultured primary rat hepatocytes or assays containing subcellular fractions prepared from rat liver, arsenobetaine aldehyde is the common intermediate converted to arsenobetaine or to both trimethylarsine oxide and trimethylarsine ${ }^{[20]}$ (Fig. 3). Arsenocholineconverting activity is most enriched in mitochondria; activity for conversion of trimethylarsine oxide to trimethylarsine is most enriched in liver homogenate. Choline dehydrogenase or an equivalent mitochondrial aldehyde dehydrogenase ${ }^{[21]}$ catalyzes conversion of arsenocholine to arsenobetaine or trimethylarsine oxide. As noted above, trimethylarsine oxide is a liver carcinogen in rats. ${ }^{[10]} \mathrm{AS} 3 \mathrm{MT}$ catalyzes conversion of trimethylarsine oxide to trimethylarsine, ${ }^{[7]}$ a volatile genotoxic species. ${ }^{[22]}$ Chickens treated with arsenocholine exhale a strong garlic-like odor consistent with trimethylarsine expiration; ${ }^{[23]}$ thus, the chicken orthologue of mammalian AS3MT gene (J. Li et al., in preparation) may catalyze the final reductive step in this pathway.

\section{Arsenosugars}

Arsenosugars are a group of arsenic-containing carbohydrates present in a variety of marine organisms. These compounds are probably produced by symbiotic microorganisms. Their presence in higher organisms is probably related to consumption of organisms that contain arsenosugars. ${ }^{\text {[24] }}$ Exposure to arsenic through consumption of arsenosugar-rich foods can be significant. Typical Japanese diets including 20 to $30 \mathrm{~g}$ of seaweed can provide 100 to $500 \mu \mathrm{g}$ of arsenic per day ${ }^{[25]}$ In volunteers consuming arsenosugar-rich seaweed, urinary dimethylarsenic concentrations increase sharply within a day of exposure. ${ }^{[26-28]}$ In seaweed-eating sheep from Orkney Island, Scotland, daily consumption of arsenic from arsenosugars ranges from 45 to $90 \mathrm{mg}$. Dimethylarsenic is the major arsenic-containing species in urine in these sheep. ${ }^{[29,30]}$ Other metabolites identified in urine of these sheep are dimethylarsinoyl acetate and dimethylarsinothioyl acetate ${ }^{[31]}$ (Fig. 4). (The origin and significance 




Fig. 3. Scheme for conversion of arsenocholine to arsenobetaine or trimethylated arsenicals. Conversion to arsenobetaine aldehyde intermediate (Reaction 1) is catalyzed by choline oxidase or equivalent oxidase. Conversion of aldehyde to trimethylarsine oxide (Reaction 2) or to arsenobetaine (Reaction 3) is probably catalyzed by choline oxidase. Conversion of trimethylarsine oxide to trimethylarsine (Reaction 4) is possibly catalyzed by arsenic(III) methyltransferase.

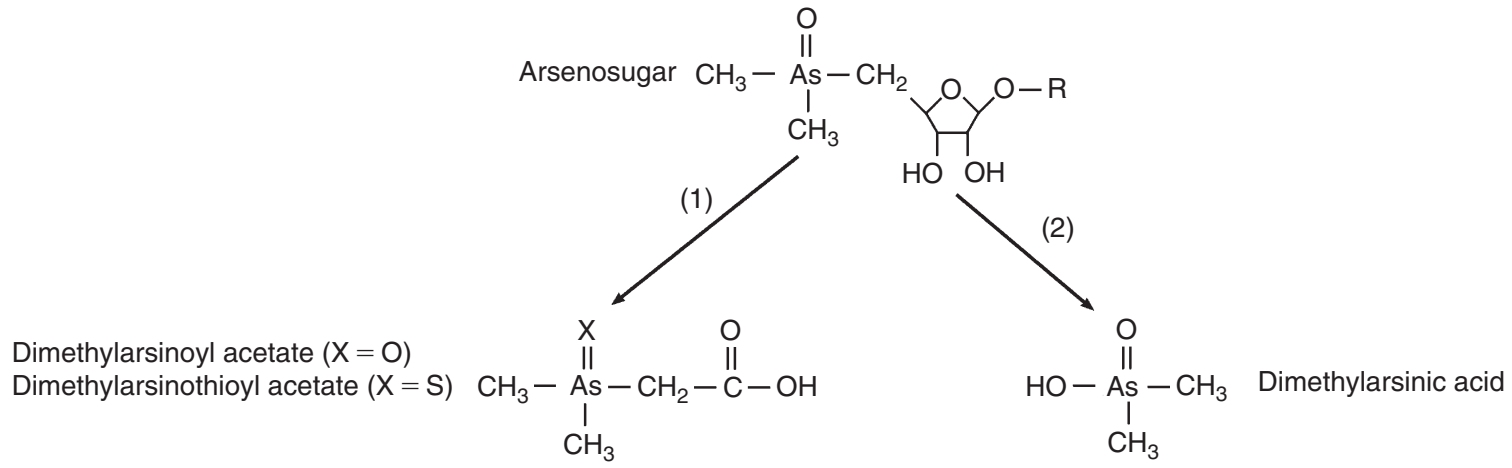

Fig. 4. Products from in vivo metabolism of arsenosugars in mammals. Urinary metabolites include dimethylarsinoyl acetate/dimethylarsinothioyl acetate and dimethylarsinic acid.

of thioylated metabolites are considered below.) Because dimethylarsinic acid is a complete carcinogen in the rat, ${ }^{[9]}$ its production from arsenosugars could be an important consideration in evaluating the risk associated with consumption of arsenosugar-rich foods.

The pathway leading from arsenosugars to dimethylarsenic and other methylated metabolites is not well understood. One possibility is that these sugars are degraded by gastrointestinal microflora, yielding metabolites that are absorbed by the mammalian host. These metabolites could undergo additional transformation or be excreted unmodified. The possibility of metabolism in the gastrointestinal tract was examined by comparing urinary metabolites in Orkney sheep adapted to a grass diet before consumption of arsenosugar-rich seaweed with those found in sheep fed exclusively seaweed ${ }^{[30]}$ Predominance of dimethylarsenic as a urinary metabolite in both groups suggests that modifications in gastrointestinal microflora induced by changes in diet did not influence metabolism of arsenosugars. Preliminary studies using anaerobic microflora of mouse intestinal caecum found that arsenosugars in seaweed extracts were degraded to dimethylarsenic (D. J. Thomas, W. R. Cullen, V. W. Lai, unpublished). Other studies found that anaerobic microflora of mouse intestinal caecum thioylate arsenosugars (S. D. Conklin, J. T. Creed, D. J. Thomas, in preparation). Taken together, these results suggest that gastrointestinal tract microorganisms could play roles in conversion of arsenosugars to methylated metabolites. 


\section{Problems and Prospects for Future Research}

As shown above, exposure to a diverse group of arsenicals leads to production of methylated metabolites that have been implicated as mediators of toxic and carcinogenic effects of arsenic. Although the pathway for iAs metabolism has been partially elucidated, less is known about processes that convert trimethylarsonium compounds and arsenosugars to methylated species. Unravelling these processes will require use of standard techniques for the study of xenobiotic metabolism in cells and organisms and application of analytical techniques designed to identify and quantify each arsenic-containing species. Two specific issues have emerged in recent years that must be addressed in future analytical studies. First, as noted above, the oxidation state of arsenic in various metabolites affects their toxic potency. ${ }^{[11]}$ Thus, determination of the oxidation state of arsenic would provide insight into metabolic processes underlying conversion of arsenicals. Although $\mathrm{pH}$-selective hydride-generation AAS of arsenicals permits quantitation by oxidation state, ${ }^{[32,33]}$ this labour-intensive method requires sample collection under conditions that preserve the oxidation state of arsenic. Alternative methods involving solid-phase extraction of biological samples may improve accuracy of oxidation state determination. Second, identification of thioylated arsenicals such as dimethylarsinothioyl acetate (Fig. 4) or thioarsenosugars ${ }^{[34]}$ has raised new analytical challenges and posed new questions about metabolism. For instance, arsinothioyls confound identification by HPLC, $\mathrm{pH}$-selective HG-ICP-MS, or anionexchange chromatography. ${ }^{[35,36]}$ Formation in vitro or in vivo of thioylated metabolites by rat liver ${ }^{[37,38]}$ may require enzymatic generation of hydrogen sulfide. ${ }^{[39]}$ Understanding formation of arsinothioyls and their biological activities will provide more information about roles of metabolism in the actions of arsenicals as toxins and carcinogens.

\section{Acknowledgments}

We thank our colleagues for advice and discussion on the topics covered in this manuscript. S.B.W. was supported by Training Grant T901915 of the USA Environmental Protection Agency - University of North Carolina Toxicology Research Program. V.D. was supported by a MECD/Fulbright Grant for Postdoctoral Training (Ministry of Education, Culture, and Sports, Spain). Z.D. and M.S. are supported by NIH grants ES010845 and DK 56350. This manuscript has been reviewed in accordance with the policy of the USA Environmental Protection Agency and approved for publication. Approval does not signify that contents necessarily reflect the views and policies of the Agency, nor does mention of trade names or commercial products constitute endorsement or recommendation for use.

\section{References}

[1] T. Yoshida, H. Yamauchi, G. F. Sun, Toxicol. Appl. Pharmacol. 2004, 198, 243. doi:10.1016/J.TAAP.2003.10.022

[2] A. H. Smith, E. O. Lingas, M. Rahman, Bull. World Health Organ. 2000, 78, 1093.

[3] A. A. Maherg, Venomous Earth 2005 (Macmillan: New York, NY).
[4] B. Nemeti, Z. Gregus, Toxicol. Sci. 2005, 85, 847. doi:10.1093/ TOXSCI/KFI157

[5] Z. Gregus, B. Nemeti, Toxicol. Sci. 2005, 85, 859. doi:10.1093/ TOXSCI/KFI158

[6] S. B. Waters, V. Devesa, L. M. Del Razo, M. Styblo, D. J. Thomas, Chem. Res. Toxicol. 2004, 17, 404. doi:10.1021/TX0342161

[7] S. B. Waters, V. Devesa, M. W. Fricke, J. T. Creed, M. Styblo, D. J. Thomas, Chem. Res. Toxicol. 2004, 17, 1621. doi:10.1021/ TX0497853

[8] Z. Drobna, S. B. Waters, V. Devesa, A. W. Harmon, D. J. Thomas, Toxicol. Appl. Pharmacol. 2005, 207, 147. doi:10.1016/J.TAAP.2004.12.007

[9] M. Wei, H. Wanibuchi, S. Yamamoto, W. Li, S. Fukushima, Carcinogenesis 1999, 20,1873. doi:10.1093/CARCIN/20.9.1873

[10] J. Shen, H. Wanibuchi, E. I. Salim, M. Wei, A. Kinoshita, K. Yoshida, G. Endo, S. Fukushima, Carcinogenesis 2003, 24 1827. doi:10.1093/CARCIN/BGG143

[11] D. J. Thomas, M. Styblo, S. Lin, Toxicol. Appl. Pharmacol. 2001, 176, 127. doi:10.1006/TAAP.2001.9258

[12] A. W. Ritchie, J. S. Edmonds, W. Goessler, R. O. Jenkins, FEMS Microbiol. Lett. 2004, 235, 95. doi:10.1016/J.FEMSLE. 2004.04.016

[13] J. Fujihara, T. Kunito, R. Kubota, S. Tanabe, Comp. Biochem. Physiol C: Toxicol. Pharmacol. 2003, 136, 287. doi:10.1016/J.CCA.2003.10.001

[14] L. A. Clowes, K. A. Francesconi, Comp. Biochem. Physiol. C: Toxicol. Pharmacol. 2004, 137, 35. doi:10.1016/J.CCA.2003. 11.003

[15] R. O. Jenkins, A. W. Ritchie, J. S. Edmonds, W. Goessler, N. Molenat, D. Kuehnelt, C. F. Harrington, P. G. Sutton, Arch. Microbiol. 2003, 180, 142. doi:10.1007/S00203-003-0569-9

[16] P. J. G. Mann, H. E. Woodward, J. H. Quastel, Biochem. J. 1938, 32, 1024

[17] M. Vahter, E. Marafante, L. Dencker, Sci. Total Environ. 1983, 30, 197. doi:10.1016/0048-9697(83)90012-8

[18] E. Marafante, M. Vahter, L. Dencker, Sci. Total Environ. 1984, 34, 223. doi:10.1016/0048-9697(84)90065-2

[19] R. Heinrich-Ramm, S. Mindt-Prufert, D. Szadkowski, J. Chromatogr. 2002, 778, 263. doi:10.1016/S0378-4347(01)00443-1

[20] A. Christakopoulos, H. Norin, M. Sandstrom, H. Thor, P. Moldeus, R. Ryhage, J. Appl. Toxicol. 1988, 8, 119.

[21] M. K. Chern, R. Pietruszko, Biochem. Cell Biol. 1999, 77, 179. doi:10.1139/BCB-77-3-179

[22] P. Andrewes, K. T. Kitchin, K. Wallace, Chem. Res. Toxicol. 2003, 16, 994. doi:10.1021/TX034063H

[23] T. H. Jukes, A. D. Welch, J. Biol. Chem. 1942, 146, 19.

[24] U. Nørum, V. W. Lai, S. A. Pergantis, W. R. Cullen, J. Environ. Monit. 2005, 7, 122. doi:10.1039/B412311E

[25] H. Yamauchi, K. Takahashi, M. Mashiko, J. Saithoh, Y. Yamamura, Appl. Organometal. Chem. 1992, 6, 383. doi:10.1002/AOC.590060412

[26] X. C. Le, W. R. Cullen, K. J. Reimer, Clin. Chem. 1994, 40,617

[27] M. Ma, X. C. Le, Clin. Chem. 1998, 44, 539.

[28] K. A. Francesconi, R. Tanggaar, C. J. McKenzie, W. Goessler, Clin. Chem. 2002, 48, 92.

[29] J. Feldmann, K. John, P. Pengprecha, Fresenius J. Anal. Chem 2000, 368, 116. doi: $10.1007 / \mathrm{S} 002160000482$

[30] H. R. Hansen, A. Raab, K. A. Francesconi, J. Feldmann, Environ Sci. Technol. 2003, 37, 845. doi:10.1021/ES026074N

[31] H. R. Hansen, R. Pickford, J. Thomas-Oates, M. Jaspers, J. Feldmann, Angew. Chem. Int. Ed. 2004, 43, 337. doi:10.1002/ ANIE. 200352740

[32] L. M. Del Razo, M. Styblo, W. R. Cullen, D. J. Thomas, Toxicol. Appl. Pharmacol. 2001, 174, 282. doi:10.1006/ TAAP.2001.9226

[33] V. Devesa, L. M. Del Razo, B. Adair, Z. Drobna, S. B. Waters, M. F. Hughes, M. Styblo, D. J. Thomas, J. Anal. At. Spectrom. 2004, 19, 1460. doi:10.1039/B407388F 
[34] E. Schmeisser, R. Raml, K. A. Francesconi, D. Kuehnelt, A. L. Lindberg, C. Soros, W. Goessler, Chem. Commun. 2004, 21, 1824. doi:10.1039/B406917J

[35] H. R. Hansen, A. Raab, M. Jaspars, B. F. Milne, J. Feldmann, Chem. Res. Toxicol. 2004, 17, 1086. doi:10.1021/ TX049978Q

[36] J. A. Jay, N. K. Blute, H. F. Hemond, J. L. Durant, Water Res. 2004, 38, 1155. doi:10.1016/J.WATRES.2003.11.014
[37] H. R. Hansen, M. Jaspars, J. Feldmann, Analyst 2004, 129, 1058. doi:10.1039/B409661B

[38] K. T. Suzuki, B. K. Mandal, A. Katagiri, Y. Sakuma, A. Kawakami, Y. Ogra, K. Yamaguchi, Y. Sei, K. Yamanaka, K. Anzai, M. Ohmichi, H. Takayama, N. Aimi, Chem. Res. Toxicol. 2004, 17, 914. doi:10.1021/TX049963S

[39] K. Eto, H. Kimura, J. Biol. Chem. 2002, 277, 42680. doi:10.1074/ JBC.M205835200 